\title{
AOTrauma Master Course Hand and Wrist
}

\author{
Joachim Windolf, Wolfgang Linhart
}

\begin{abstract}
Am 16. und 17.03.2017 fand der 1. AOTrauma Master Course Hand and Wrist in Deutschland statt. Unter der Leitung von Prof. J. Windolf, Prof. W. Linhart und Dr. W. Schäfer wurde den 40 Teilnehmern aus aller Welt ein Intensivprogramm aus dem Bereich der Handchirurgie geboten.
\end{abstract}

In Anlehnung an die jährlich stattfindenden AOTraumaHandkurse wurde besonderes Gewicht auf den praktischen Teil des Kurses an anatomischen Präparaten gelegt. Im anatomischen Institut der Universität Düsseldorf (Leiter: Prof. T. Filler) fanden sich dafür sehr gute Bedingungen. Durch die besondere Fixierung der Präparate ist das Gewebe dem „Natürlichen“ sehr ähnlich, sodass die Übungen sehr realitätsnah erfolgen konnten.

Neben den praktischen Übungen wurde den Teilnehmern im theoretischen Teil in Vorträgen über ausgewählte zentrale Themen Expertenwissen vermittelt und die Gelegenheit für eine intensive Diskussion gegeben. Die Kursleitung wurde von mehr als 10 anerkannten Experten der Handchirurgie unterstützt, sodass vor allem in den praktischen Übungen eine individuelle Betreuung der 40 Teilnehmer gewährleistet war. Neben den Vorträgen wurden begleitend Fälle demonstriert, was die offene Diskussion sehr belebte und den Erfahrungsaustausch anstieß.

Der 1. Block des Kurses behandelte intraartikuläre Frakturen der Hand, während im 2. Block die intraartikuläre distale Radiusfraktur im Mittelpunkt stand. Hierfür standen erstmals Präparate mit einer artifiziell erzeugten distalen Radiusfraktur, in der Regel Typ 23-C3, bereit. Begleitend hierfür lagen den Teilnehmern Computertomografien des Handgelenks individuell passend zum Präparat vor. Somit konnte sowohl die Herangehensweise und Planung als auch die Osteosynthese mit modernen winkelstabilen Implantaten unter professioneller Anleitung geübt werden. Eine radiologische Kontrolle der Ergebnisse fand im Präparationsaal statt. Der 3. Block des Kurses befasste sich mit Begleitverletzungen des distalen radioulnaren Gelenks und der Bandverletzungen der angrenzenden Handwurzelgelenke. Weichteilproblematiken durch Komplexverletzungen sowie Infektionen fanden sich im 4. Block des Kurses. Die praktischen Übungen zu diesem Thema umfassten lokale Lappenplastiken an der Hand und am Unterarm.

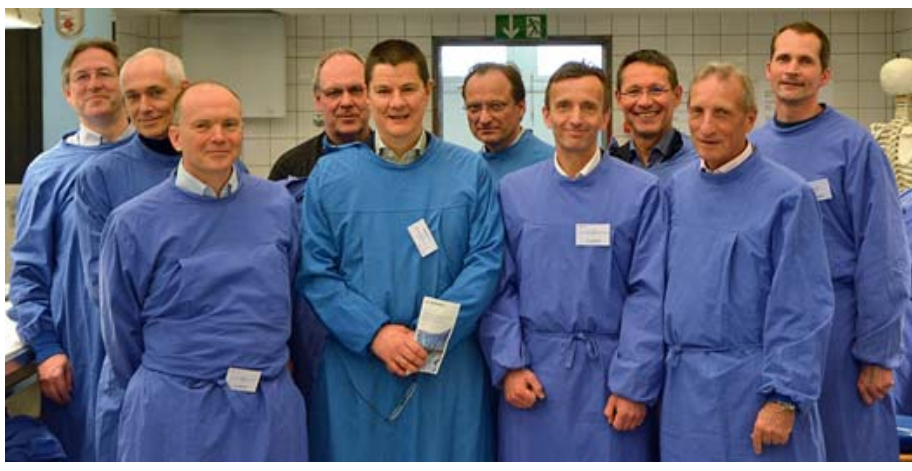

Abb. 1 Faculty des Kurses. Bild: Prof. Windolf.

Der persönliche Kontakt und Austausch wurde durch ein gemeinsames Abendessen in Düsseldorf am Abend des 1. Kurstages vertieft. Das Feedback am Kursende durch die Teilnehmer war deutlich positiv, neben einem intensiven fachlichen Austausch wurde die hohe Vortragsqualität und rege Diskussion in guter Atmosphäre gelobt.

\section{Korrespondenzadresse}

Prof. Dr. Joachim Windolf

Düsseldorf

windolf@uni-duesseldorf.de

Prof. Dr. Wolfgang Linhart

Heilbronn

wolfgang.linhart@slk-kliniken.de

Bibliografie

DOI https://doi.org/10.1055/s-0043-116391

OP-JOURNAL 2017; 33: 197 @ Georg Thieme Verlag KG Stuttgart · New York ISSN 0178-1715 